\title{
Oxygen saturation in cystic fibrosis
}

\author{
M Betancourt, G Slade, R Dinwiddie
}

\begin{abstract}
The availability of non-invasive oxygen saturation $\left(\mathrm{SaO}_{2}\right)$ measurement could prove to be a useful tool for following up the progress of patients with cystic fibrosis. The present study was undertaken to compare its use with other routine measurements in the clinic. A total of 100 patients with cystic fibrosis were compared with 50 patients with stable asthma. The children were aged between 5 and 16 years and were studied as outpatients. A positive correlation was found between forced expiratory volume in one second and $\mathrm{SaO}_{2}$ in the group with cystic fibrosis and in the comparison group. Median $\mathrm{SaO}_{2}$ in those with cystic fibrosis $(94.0 \%)$ was significantly lower than in controls $(97 \cdot 0 \%)$. Significant correlations were also found in the patients with cystic fibrosis for the following: ShwachmanKulczycki score, higher weight centiles, and chronic pseudomonas lung infection with or without staphylococci. An inverse correlation was found with the Chrispin-Norman chest $x$ ray score. $\mathrm{SaO}_{2}$ measurements are useful in the outpatient assessment of patients with cystic fibrosis and compare well with other standard tests of disease severity.
\end{abstract}

Pulse oximetry is a reliable and simple method for assessing oxygen status in children at all ages. ${ }^{1}$ It is non-invasive, easy to apply, and provides rapid results. The aim of this study was to assess oxygen saturation $\left(\mathrm{SaO}_{2}\right)$ in children with cystic fibrosis and to relate this to the patient's general condition and other specific measurements of clinical status.

\section{Patients and methods}

One hundred children with cystic fibrosis consecutively attending the outpatient clinic and 50 comparison patients with asthma, which was well controlled on routine medication, were included in this study over a six month period. They were aged between 5 and 16 years.

$\mathrm{SaO}_{2}$ was measured using an Ohmeda Biox 3700 pulse oximeter with a paediatric finger probe before the other lung function measurements. The saturation measurements were made after allowing the readings to stabilise; this usually took two to three minutes with a minimum time of 30 seconds. Lung function was assessed using a Vitalograph Compact spirometer to measure forced vital capacity (FVC), forced expiratory volume in one second $\left(\mathrm{FEV}_{1}\right)$, peak expiratory flow rate (PEFR), and forced expiratory flow at $75 \%$ of expired FVC $\left(\mathrm{FEF}_{75 \%}\right)$.

The clinical evaluation of the patients with cystic fibrosis was based on the ShwachmanKulczycki score ${ }^{2}$ utilising general activity, physical examination, nutrition, and findings on radiography (scoring system of $0-100 ; 100=$ excellent, $\angle 40=$ severe disease). Radiological assessment was undertaken blind by a physician experienced in cystic fibrosis (RD) and used the Chrispin-Norman score. ${ }^{3}$ Chronic lung infection was considered to be the presence of the same pathogen in three successive sputum cultures over a six month period.

The lung function test results were expressed as percentage of predicted normal for height using reference values from the Brompton Hospital study, 1985 (unpublished observations). These values were obtained on 788 normal local schoolchildren of the same age, height, and sex distribution as those in our study. These data are most representative of the local population and compare well with standard reference values. ${ }^{4}$ Weight was expressed as age centile for sex based on data obtained on normal children. Statistical analysis was by Pearson correlation and Wilcoxon rank sum test.

\section{Results}

One hundred patients with cystic fibrosis and 50 patients with stable asthma as a comparison group were studied. Thirty seven cystic fibrosis patients were aged less than 8 years, 40 were aged 8-12, and 23 were aged 12-16. The comparison group comprised 12 children aged less than 8 years, 23 aged between 8 and 12 years, and 15 aged between 12 and 16. There was a positive correlation between $\mathrm{SaO}_{2}$ and $\mathrm{FEV}_{1}$ in the cystic fibrosis patients $(r=0.5, p<0.001)$ (figure) and in the comparison group $(r=0.4$, $\mathrm{p}<0.01$ by Pearson correlation); these data were normally distributed. The median values for $\mathrm{SaO}_{2}$ were: comparison group $97 \cdot 0 \%$ (range= 94-99\%) and cystic fibrosis patients $94.0 \%$ (range $=70-99 \%, p<0.001$ ). There was a significant correlation for patients with cystic fibrosis between $\mathrm{SaO}_{2}$ and clinical evaluation using the Shwachman-Kulczycki score $(\mathbf{p}<0 \cdot 01)$. There was an inverse correlation between $\mathrm{SaO}_{2}$ and Chrispin-Norman score $(\mathrm{p}<0.05)$.

The median $\mathrm{SaO}_{2}$ difference between patients with cystic fibrosis with and without chronic lung infection was assessed using the Wilcoxon rank sum test. Cystic fibrosis patients with chronic pseudomonas infection $(n=37)$ had a median $\mathrm{SaO}_{2}$ of $92 \cdot 0 \%$ (range=75-97\%) and without infection $(n=6) 95 \cdot 5 \%$ (range $=94-97 \%$;
Correspondence to Dr Dinwiddie.

Accepted 7 February 1991 


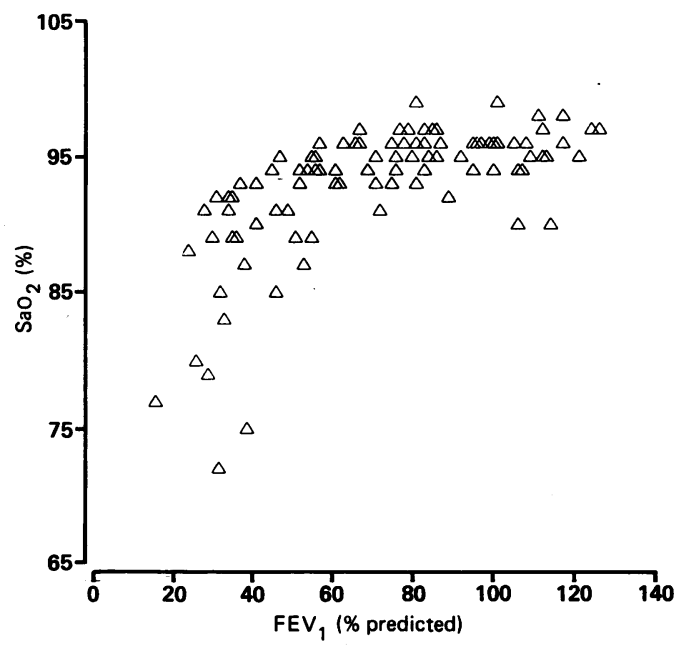

Oyxgen saturation $\left(\mathrm{SaO}_{2}\right)$ and forced expiratory volume in one second $\left(F E V_{1}\right) \%$ predicted for height in 100 patient with cystic fibrosis (includes seven overlapping points). Reproduced with permission from the Fournal of the Royal Society of Medicine Supplement 1991;84(18):36-9, figure 1. Cystic fibrosis: yesterday, today and tomorrow by $R$ Dinwiddie.

$\mathrm{p}<0.01)$. Those with chronic pseudomonas and staphylococcal lung infection combined $(n=6)$ had a median $\mathrm{SaO}_{2}$ of $93 \%$ (range $=79-96 \%$; $\mathrm{p}<0.01$ ). Patients with chronic staphylococcal lung infection only $(n=34)$ showed a higher $\mathrm{SaO}_{2}$ (median 95.0\%, range $=87-99 \%$ ) than those with pseudomonas only (median $92.0 \%$; $\mathrm{p}<0.001)$. The higher weight centiles $(>50$ th $)$ correlated well with higher $\mathrm{SaO}_{2}$ values $(>94 \%)$ for cystic fibrosis patients $(p<0 \cdot 01)$.

\section{Discussion}

There was a positive correlation between the $\mathrm{SaO}_{2}$ and \% predicted $\mathrm{FEV}_{1}$ in each patient (figure); this was not unexpected. In cystic fibrosis patients, lung function deteriorates because of bacterial colonisation resulting in persistent infection and secondary airway obstruction.

As expected, higher $\mathrm{SaO}_{2}$ levels correlated well with good clinical status and low radiological scores. In patients with cystic fibrosis with better weight gain, there is also a higher $\mathrm{SaO}_{2}$. Poor nutritional state is related to decreased resistance to infection with subsequent decline in pulmonary function.

\section{CONCLUSIONS}

$\mathrm{SaO}_{2}$ measurements are a useful addition to the clinical and lung function assessment of cystic fibrosis patients. They correlate well with clinical status and other more specific methods of assessment including radiology and nutrition. Although they do not replace basic measurements with less complicated apparatus such as the spirometer, they do represent the final common pathway of oxygen delivery to the tissues. Although not recommending that every cystic fibrosis clinic should have an oximeter, the ability to utilise such measurements either individually or longitudinally will become increasingly valuable in the future care and management of these patients.

We thank Miss Angie Wade for her expert statistical advice, Aidan Laverty for his invaluable technical assistance, and Paula Smith for typing the manuscript.

1 Cote CJ, Goldstein EA, Cote MA, Hoaglin DC, Ryan JF. A single-blind study of pulse oximetry in children. AnesA single-blind study of puls

2 Shwachman H, Kulczycki LL. Long-term study of one hundred five patients with cystic fibrosis. Am $\mathcal{J}$ Dis Child hundred five pati

3 Chrispin AR, Norman AP. The systematic evaluation of the chest radiograph in cystic fibrosis. Pediatr Radiol 1974;2: $101-6$

4 Polgar G, Weng TR. The functional development of the respiratory system. Am Rev Resp Dis 1979;120:625-95. 